\title{
Zum Verständnis der unendlichen Energieeigenwerte, die in der MO-LCAO-Methode mit teilweiser Uberlappung auftreten
}

\author{
Von W. A. Bingel, H. Preuss, \\ Max-Planck-Institut für Physik und Astrophysik, München, \\ L. HoFaCKer, \\ Institut für physikalische Chemie der Universität Göttingen, \\ und H.-H. SchmidtKe, \\ Cyanamid European Research Institute, Cologny, Genf \\ (Z. Naturforschg. 16 a, 1334-1338 [1961] ; eingegangen am 7. Oktober 1961)
}

\begin{abstract}
It is known that the MO-LCAO-method with partial inclusion of overlap can lead to infinite energy values. The reason for this physically impossible behaviour is investigated here. It is shown, that the infinite energy values are a consequence of the inconsistent amputation of the overlap and energy matrix. Methods of avoiding this difficulty are discussed. The general theory is illustrated by application to the allyl radical.
\end{abstract}

Bei der theoretischen Behandlung konjugierter organischer Molekeln nach der MO-LCA.O-Methode sind bisher folgende Näherungsstandpunkte eingenommen worden:

1. Von HǘKel ${ }^{1}$, der als erster die MO-Methode in die theoretische Chemie der konjugierten Verbindungen eingeführt hat, wird als Überlappungsmatrix $\Im$ der Atomfunktionen die Einheitsmatrix gewählt. In der Matrix $\mathfrak{S}$ des Hamilton-Operators $H$ werden dagegen Wechselwirkungen mit den nächsten Nachbarn, aber keine weiteren berücksichtigt. Die Eigenwerte der Energie haben im Falle gleichatomiger Systeme die Form

$$
E_{i}=\alpha+\beta \varrho_{i},
$$

wo $\alpha$ und $\beta$ Elemente der Ş-Matrix sind, die als Couloмв- bzw. Resonanzintegral bezeichnet werden. Die $\varrho_{i}$ sind aus der Geometrie der Molekeln bestimmte reine Zahlen.

2. Wheland ${ }^{2}$ hat die Hückelsche Methode später modifiziert, indem er auch die Überlappung $S$ zwischen benachbarten Atomfunktionen in Betracht zog; dabei blieb die $\mathfrak{S}_{\text {-Matrix }}$ im Sinne Hückels unverändert. Auf diese Weise berechnet man die Energieeigenwerte $\mathrm{zu}$

$$
E\left(\varrho_{i}\right)=\left(\alpha+\beta \varrho_{i}\right) /\left(1+S \varrho_{i}\right) .
$$

1 E. Hückel, Z. Phys. 60, 423 [1930]; 70, 204 [1931]; 72, 310 [1931]; 76, 628 [1932].

2 G. W. Wheland, J. Amer. Chem. Soc. 63, 1770 [1941].

3 H. Hartmann, Z. Naturforschg. 15 a, 993 [1960].

4 W. A. Bingel, H. Preuss u. H.-H.Schmidtre, Z. Naturforschg. 16 a, 1328 [1961], voranstehend.
3. Hartmann ${ }^{3}$ hat vor kurzem die Hückelsche Theorie so erweitert, daß er neben den $2 \mathrm{p} \pi$-Funktionen auch höhere Atomfunktionen einführte. In entsprechender Weise ergeben sich auch hier die Eigenwerte der Energie als Funktion der gleichen strukturabhängigen Parameter $\varrho_{i}$.

4. Schließlich läßt sich auch die Hartmannsche Näherung durch Mitnahme der Überlappung zwischen nächsten Nachbarn noch vervollständigen ${ }^{4}$, . Man erhält auch hier Energieeigenwerte als Funktion der bekannten $\varrho_{i}$.

Es läßt sich zeigen, daß der Wertbereich der möglichen $\varrho_{i}$

$$
-3 \leq \varrho_{i} \leq+3
$$

ist $^{5}$. Nach Gl. (2) kann es daher vorkommen, daß für einen bestimmten Wert der Überlappung $\left[S=-\left(1 / \varrho_{i}\right)\right]$ ein unendlicher Energiewert auftritt. Diese Schwierigkeit ergibt sich immer dann, wenn die Überlappung in unvollständiger Weise (etwa Beschränkung auf nächste Nachbarn) eingeführt worden ist. In der Näherung des Punktes 4. sind diese Singularitäten der Energie besonders schwerwiegend, weil die Überlappungsintegrale zwischen höheren Atomfunktionen viel näher an 1 liegen als es bei den 2p $\pi$-Funktionen der Hückelschen Theorie der Fall ist.

\footnotetext{
* Wie wir inzwischen erfahren haben, hat auch Herr Dr. G. Gliemann (Institut für physikalische Chemie der Universität Frankfurt/Main) zur gleichen Zeit ähnliche Untersuchungen durchgeführt, ohne sie zu publizieren.

5 C. A. Coulson, Proc. Camb. Phil. Soc. 46, 204 [1949].
} 
Solange dieser Widerspruch nicht beseitigt ist, müssen alle Berechnungen von konjugierten Systemen mit teilweiser Überlappung in Zweifel gezogen werden. Keinesfalls darf man erwarten, daß die unkritische Mitnahme von gewissen Überlappungen eine Verbesserung bedeutet. Aufgabe dieser Arbeit ist es, eine Erklärung für das Auftreten der Singularitäten zu geben und die Möglichkeiten zu ihrer Vermeidung zu prüfen.

\section{Die Natur der Singularitäten}

Zum besseren Verständnis des vorliegenden Problems wollen wir zunächst einige einfache Zusammenhänge zwischen der linearen Abhängigkeit eines Funktionssatzes und der Größe der zugehörigen Determinanten von Überlappungs- und Energiematrix anführen.

Bekanntlich besteht die notwendige und hinreichende Bedingung für die lineare Abhängigkeit eines Funktionssatzes $\varphi_{i}(i=1, \ldots, N)$ darin, daß die Determinante $\left|L_{i k}\right|$ der Elemente $\left\langle\varphi_{i}|L| \varphi_{k}\right\rangle$ verschwindet, wobei $L$ ein linearer und hermitescher Operator sei.

Nehmen wir nun weiter an, daß ein linear-unabhängiger Funktionssatz in solcher Weise linear abhängig werde, daß die Funktion $\varphi_{1}$ stetig gegen eine bestimmte Linearkombination

$$
\hat{\varphi}=\sum_{j=2}^{N} b_{j}(0) \varphi_{j}
$$

der übrigen Funktionen geht, so kann weiter, ohne Einschränkung der Allgemeinheit, angenommen werden, daß dieser Übergang mittels eines reellen Parameters $p$ geschieht;

$$
\lim _{p \rightarrow 0} \varphi_{1}(p)=\hat{\varphi} .
$$

Wir wollen nun das Verhalten der Überlappungs-

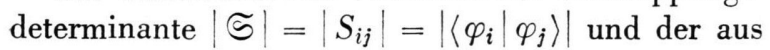
den Elementen $\left\langle\varphi_{i}|H| \varphi_{j}\right\rangle=H_{i j}$ gebildeten Determinante beim Vorgang (5) näher untersuchen.

Dazu erweitern wir $\varphi_{2}, \ldots, \varphi_{N}$ zu einem vollständigen Funktionssystem, indem wir weitere Funktionen $\varphi_{N+1} \ldots$ hinzunehmen, die wir orthogonal untereinander und auf $\varphi_{2}, \ldots, \varphi_{N}$ annehmen können. Dann läßt sich $\varphi_{1}$ nach den $\varphi_{k}(k=2,3, \ldots)$ entwickeln und wir haben

$$
\varphi_{1}=\sum_{j=2}^{N} b_{j}(p) \varphi_{j}+\sum_{j=N+1}^{\infty} b_{j}(p) \varphi_{j} .
$$

Dabei gilt

$$
\lim _{p \rightarrow 0} b_{j}(p)=\left\{\begin{array}{cl}
b_{j}(0), & j=2, \ldots, N, \\
0, & j=N+1 \ldots .
\end{array}\right.
$$

Für kleine $p$ kann Gl. (6) wegen (6a) in der Form

$$
\varphi_{1}=\hat{\varphi}+p \cdot \varphi_{1}^{\prime}+O\left(p^{2}\right)
$$

entwickelt werden. Dabei ist $\hat{\varphi}-$ definiert durch Gl. (4) - der Grenzwert für die Funktion $\varphi_{1}$ beim Ubergang zu linearer Abhängigkeit. Der zweite mit $p$ verschwindende Anteil entsteht aus dem zweiten Glied von (6) und ist nach Voraussetzung orthogonal auf $\hat{\varphi}$. Bilden wir unter Berücksichtigung von (7) und (4) die Determinanten $|S|$ und $|H|$, so nehmen sie für hinreichend kleine $p$ die folgende Form an:

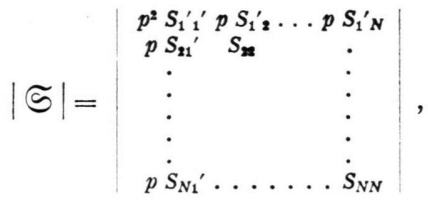

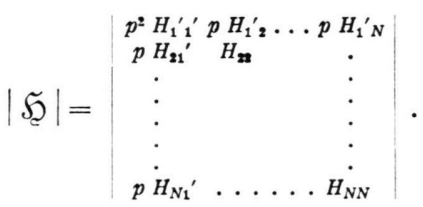

Aus (8) folgt nun, daß $|\Im|$ sowie auch $|\mathfrak{S}|$ proportional mit $p^{2}$ für $p \rightarrow 0$ gegen Null gehen. Das Säkularproblem

$$
\mathfrak{S c}=\widetilde{C} \mathfrak{C}
$$

gibt jetzt nach (8) die Säkulardeterminante

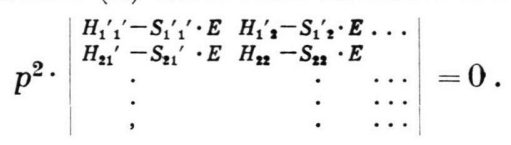

Hieraus und aus Gl. (7) ergibt sich, daß die $N$ Eigenwerte des ursprünglichen Säkularproblems (9) bei dem Grenzübergang (5) bzw. (6 a) in $N$ endliche Grenzwerte übergehen, die sich schon aus $\mathrm{Gl}$. (9 a) bei kleinem $p^{2}>0$ berechnen lassen. Dies zeigt, daß durch das Linearabhängigwerden von $\varphi_{1}$ der Grad des Säkularproblems nicht von $N$ auf $N-1$ erniedrigt wird, wie man zunächst erwarten würde, wenn der Grenzübergang wie hier vorgenommen wird. Vielmehr tritt jetzt an Stelle von $\varphi_{1}$ die neue Funktion $\varphi_{1}^{\prime}$, die sich gemäß (7) aus

$$
\varphi_{1}^{\prime}=\lim _{p \rightarrow 0}\left(\frac{\varphi_{1}-\hat{\varphi}}{p}\right)
$$

bestimmt. 
Ein solches Verhalten des Funktionssatzes ist in einem anderen Zusammenhang schon früher beobachtet worden. So zeigt sich, daß der "open-shell“" A.nsatz

$$
3 \Psi=N^{\prime}\left[1 \mathrm{~s}(1) 1 \mathrm{~s}^{\prime}(2)-1 \mathrm{~s}(2) 1 \mathrm{~s}^{\prime}(1)\right]
$$

für den 1s $2 \mathrm{~s}^{3} \mathrm{~S}$-Zustand des He-Atoms beim Übergang zum „closed-shell“-Fall $\left(1 \mathrm{~s}^{\prime} \rightarrow 1 \mathrm{~s}\right)$ nicht verschwindet, sondern in

$$
N[1 \mathrm{~s}(1) 2 \mathrm{~s}(2)-1 \mathrm{~s}(2) 2 \mathrm{~s}(1)]
$$

übergeht ${ }^{6}$, wobei die beiden Terme in ${ }^{3} \Psi$ den Funktionen $\varphi_{1}$ und $\hat{\varphi}$, der Ausdruck ( $7 \mathrm{~b}$ ) der Funktion $\varphi_{1}^{\prime}$ in Gl. (7) entsprechen.

Ein anderes Beispiel ist die MO-LCAO-Berechnung des $\sigma_{\mathfrak{u}}$-Zustandes

$$
\sigma_{\mathfrak{u}}=\left\{2\left(1-S_{\mathrm{ab}}\right)\right\}^{-1 / 2} \cdot\left(1 \mathrm{~s}_{\mathrm{b}}-1 \mathrm{~s}_{\mathrm{a}}\right)
$$

des $\mathrm{H}_{2}{ }^{+}$-Moleküls.

Für $R_{\mathrm{ab}} \rightarrow 0$ verschwindet die Funktion ( $7 \mathrm{c}$ ) nicht, sondern geht in die Funktion $1 \mathrm{p} \sigma_{\mathrm{a}}$ über, die wieder mit $\varphi_{1}^{\prime}$ von Gl. (7 a) zu identifizieren ist. Ein entsprechender Grenzübergang für zwei $2 \mathrm{p} \tau$-Funktionen liefert für verschwindenden Kernabstand $R_{\mathrm{ab}}$ eine Funktion vom Typus $2 \mathrm{~d} \pi$. In allen Fällen bleiben alle Eigenwerte der Säkulargleichung (9) beim Grenzübergang (5) endlich.

Aus diesen Überlegungen folgt nun weiter, daß bei konsequenter Durchführung der MO-LCAOMethode, die ja auf ein Säkularproblem (9) führt, kein Unendlichwerden der Energie auftreten kann, solange die Elemente der Determinanten $|\Im|$ und $|\mathfrak{S}|$ mit dem tatsächlich vorliegenden Funktionssystem berechnet werden, welches hier aus den Atomfunktionen $\Phi_{\mu}$ besteht, die bei den jeweiligen Atomen des Moleküls zentriert sind.

Die Molekülfunktionen $\psi_{i}$ sind dann

$$
\psi_{i}=\sum_{\mu=1}^{N} c_{\mu i} \Phi_{\mu} ; \quad i=1,2, \ldots, N,
$$

wobei die Koeffizienten $c_{\mu i}$ zu der Matrix c zusammengefaßt seien. Die $\psi_{i}$ und $\Phi_{\mu}$ können ebenfalls zu je einem Zeilenvektor

$$
\psi=\left(\psi_{1}, \psi_{2}, \ldots, \psi_{N}\right) ; \quad \boldsymbol{\Phi}=\left(\Phi_{1}, \Phi_{2}, \ldots, \Phi_{N}\right)
$$

zusammengefaßt werden, womit sich die $N$ Gleichungen (10) in der Matrixform

ergeben.

$$
\psi=\boldsymbol{\Phi} \cdot \mathrm{c}
$$

${ }^{6}$ H. Shull u. P. O. Löwdin, J. Chem. Phys. 25, 1035 [1956].

7 P. O. Löwdin, Phys. Rev. 97, 1447 [1955].
Durch die Substitution $^{7}$

$$
\mathrm{c}=\Im^{-1 / 2} \cdot \mathrm{c}^{\prime}
$$

geht die Säkulargleichung (9) über in

$$
\mathfrak{S g}^{\prime} \mathfrak{c}^{\prime}=\mathfrak{c}^{\prime} \mathfrak{F} \text {, }
$$

wobei

$$
\mathfrak{S}^{\prime}=\mathfrak{S}^{-1 / 2} \mathfrak{S} \mathbb{S}^{-1 / 2}
$$

ist. Aus (10 a) und (11) folgt

$$
\boldsymbol{\psi}=\boldsymbol{\Phi} \widetilde{\varsigma}^{-1 / 2} \cdot c^{\prime}=\boldsymbol{\varphi} \cdot c^{\prime} .
$$

Dabei sind die zu dem Zeilenvektor $\psi$ zusammengefaßten $\varphi_{\mu}$ zueinander orthogonal.

Da nun bekanntlich das Produkt aller Eigenwerte $E_{i}$ von (12) durch

$$
\Pi E_{i}=\left|\mathfrak{S}^{\prime}\right|=|\mathfrak{S}| /|\Im|
$$

gegeben ist, folgt, daß nur dann unendliche Energie-

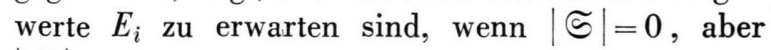
$|\mathfrak{S}| \neq 0$ bleibt. Dies kann nach Gl. (8) und folgendem Text bei einer konsequenten Rechnung nicht vorkommen.

\section{Konsequente Formulierung der MO-LCAO- Methode mit Nachbarüberlappung}

In der naiven MO-LCAO-Methode nach HüCKEL ${ }^{1}$ und $\mathrm{W}_{\text {HeLAND }}{ }^{2}$ werden die ursprünglichen Matrizen $\mathfrak{S}$ und $\mathfrak{S}$ durch Wegstreichen von Nichtnachbargliedern vereinfacht $\mathrm{zu}$

$$
\tilde{\mathfrak{S}}=\alpha I+\beta \mathfrak{g}, \quad \widetilde{S}=I+S \mathfrak{g} .
$$

Dabei ist $\mathfrak{g}$ die topologische ${ }^{8}$ Matrix (auch Strukturmatrix ${ }^{3}$ genannt). Solch eine Amputation führt zu den schon in der Einleitung erwähnten Singularitäten ${ }^{4}$. Im Gegensatz dazu läßt sich die Form (16) für die S-Matrix in korrekter Weise durch die folgende Basistransformation $\mathfrak{U}$ erzielen

$$
\begin{aligned}
\mathfrak{U} & =\mathfrak{S}^{-1 / 2}(I+S \mathfrak{g})^{+1 / 2}, \\
\mathfrak{S}^{\prime \prime} & =\mathfrak{U}^{+} \mathfrak{S} \mathfrak{U}=\tilde{\mathbb{S}}=I+S \mathfrak{g} .
\end{aligned}
$$

Durch die gleiche Transformation geht $\mathfrak{S}$ über in

$$
\mathfrak{H}^{\prime \prime}=\mathfrak{U}^{+} \mathfrak{S} \mathfrak{U},
$$

was jedoch i. allg. von $\tilde{\mathfrak{H}}$ verschieden ist. Damit läßt sich die gleichzeitige Amputation von $\mathfrak{S}$ und $\mathfrak{S}$ gemäß Gl. (16) nicht mehr auf eine gemeinsame Basistransformation zurückführen. Dies ist die Ursache für die Singularitäten in der MO-LCAO-Methode 
mit Nachbarüberlappung. Für bestimmte Werte von $S$ erweist sich nämlich das Wegstreichen von Nichtnachbargliedern in $\subseteq$ und $\mathfrak{S}$ nach (16) in der Weise als unglücklich, daß $|\tilde{\Im}|=0$ wird, aber $|\tilde{\mathfrak{H}}| \neq 0$ bleibt [s. Gl. (15) ]. Wegen

$|\tilde{\Im}|=|I+S \mathfrak{g}|=0$ oder $|\mathfrak{g}-(-1 / S) I|=0$

hängen die kritischen Werte von $S$ mit den Eigenwerten $\varrho_{i}$ der Strukturmatrix

$$
\left|\mathfrak{g}-\varrho_{i} I\right|=0
$$

gemäß $S=-\left(1 / \varrho_{i}\right)$ zusammen [s. auch Gl. (2) ]. Aus $|\tilde{\Im}|=0$ folgt nach (17) auch $|\mathfrak{U}|=0$, was dem Übergang zu einem linear abhängigen Basissystem entspricht.

Zur Vermeidung der Singularitäten ist es daher am zweckmäßigsten, die Transformationsmatrix $\mathfrak{U}$ so zu wählen, daß immer $|\mathfrak{U}| \neq 0$ ist für alle zulässigen Werte von $S$. Im folgenden Abschnitt soll das bisher Dargelegte an einem einfachen System näher erläutert werden.

\section{Behandlung des Drei-Zentrensystems}

Wir betrachten jetzt ein System von drei gleichen Atomfunktionen (AO's) $\Phi_{\mu}(\mu=1,2,3)$ in linearer äquidistanter Anordnung. Damit haben die vollständigen $\mathfrak{S}$ - und $\widetilde{\subseteq}$-Matrizen die folgende Form:

$$
\mathfrak{S}=\left(\begin{array}{ccc}
a & \beta_{12} & \beta_{13} \\
\beta_{12} & a & \beta_{12} \\
\beta_{13} & \beta_{12} & \alpha
\end{array}\right), \quad \mathbb{S}=\left(\begin{array}{ccc}
1 & S_{12} & S_{13} \\
S_{12} & 1 & S_{12} \\
S_{13} & S_{12} & 1
\end{array}\right) \text {. }
$$

Eine einfache Transformation $\mathfrak{U}$ nach Gl. (17) ist z. B. diejenige, bei der die mittlere $\mathrm{AO} \Phi_{2}$ unverändert bleibt. Sie hat die Form

$$
\mathfrak{U}=\left(\begin{array}{lll}
a & 0 & b \\
0 & 1 & 0 \\
b & 0 & a
\end{array}\right)
$$

wobei

$$
\begin{aligned}
& a=\frac{1}{2}\left(\left(1+S_{13}\right)^{-1 / 2}+\left(1-S_{13}\right)^{-1 / 2}\right), \\
& b=-\frac{1}{2}\left(\left(1-S_{13}\right)^{-1 / 2}-\left(1+S_{13}\right)^{-1 / 2}\right) .
\end{aligned}
$$

Dabei ist $|\mathfrak{I}|=a^{2}-b^{2}=\left(1-S_{13}{ }^{2}\right)^{-1 / 2}$,

und daher immer von Null verschieden.

Mit (21) transformieren sich $\mathfrak{S}$ und $\subseteq$ nach (17) und (17 a) zu

$$
\mathfrak{S}^{\prime \prime}=\left(\begin{array}{ccc}
\frac{\alpha-\beta_{13} S_{13}}{1-S_{13}{ }^{2}} & \frac{\beta_{12}}{V 1+S_{13}} & \frac{\beta_{13}-\alpha S_{13}}{1-S_{13}{ }^{2}} \\
& a & \frac{\beta_{12}}{V 1+S_{13}} \\
& & \frac{\alpha-\beta_{13} S_{13}}{1-S_{13}{ }^{2}}
\end{array}\right),
$$

\footnotetext{
9 R. S. Mulliken, J. Chim. Phys. 46, 497 [1949].
}

$$
\widetilde{S}^{\prime \prime}=\left(\begin{array}{ccc}
1 & \frac{S_{12}}{\sqrt{1+S_{13}}} & 0 \\
& 1 & \frac{S_{12}}{V 1+S_{13}} \\
& & 1
\end{array}\right) .
$$

Wie man sieht, wird durch die Transformation die Überlappungsmatrix auf die gewünschte Form (16) gebracht. Dabei ist jedoch im Gegensatz zur amputierten Form von $\Im$, wo $S_{13}=0$ gesetzt und $S_{12}$ unverändert gelassen wird, $S_{12}$ durch den Ausdruck $S_{12} / \sqrt{1+S_{13}}$ ersetzt worden. Die Energiematrix $\tilde{\mathfrak{S}}$ dagegen hat noch nicht die Form von $\tilde{\mathfrak{F}}$ nach Gl. (16), sondern geht in diese durch die spezielle Annahme $\beta_{13}=\alpha S_{13}$ über, die mit der MulliKENschen Approximation ${ }^{9}$ in Zusammenhang steht. Die Eigenwerte haben mit dieser Annahme die Gestalt

$$
E_{ \pm}=\frac{a \pm \sqrt{2} \cdot\left(\beta_{12} / \sqrt{1+S_{13}}\right)}{1 \pm \sqrt{2} \cdot\left(S_{12} / \sqrt{1+S_{13}}\right)} \quad \text { und } \quad E_{0}=\alpha .
$$

Man ersieht aus (23) sofort, daß keiner der Energieeigenwerte unendlich werden kann, weil wegen (2l b) auch $\left|\mathbb{S}^{\prime \prime}\right| \neq 0$ bleibt und daher immer

$$
\left(S_{12} / \sqrt{1+S_{13}}\right)<1 / \sqrt{2}
$$

gilt. Soll dagegen durch die Transformation die Nachbarüberlappung $S_{12}$ unverändert bleiben, wie es bei dem Whelandschen Vorgehen der Fall ist, so ergibt sich die folgende Transformation

$$
\mathfrak{U}=\left(\begin{array}{lll}
a & c & b \\
0 & d & 0 \\
b & c & a
\end{array}\right)
$$

wo $a$ und $b$ wieder durch $(21 \mathrm{a})$ gegeben und

$$
\begin{aligned}
& c=\left(S_{12} / \sqrt{1+S_{13}}\right)(1-d), \\
& d=\sqrt{\left(1-2 S_{12}{ }^{2}\right) / \sqrt{\left(1-2 S_{12}{ }^{2}+S_{13}\right)}}
\end{aligned}
$$

sind. Dabei ist

$$
|\mathfrak{I}|=d\left(a^{2}-b^{2}\right)
$$

und kann wegen Gl. (21 b) nur für $d=0$, d. h. $S_{12}=1 / \sqrt{2}$ verschwinden. Dieser Wert kann, im Gegensatz zu (24), in der Praxis durchaus auftreten. Damit erhält das mit (25) transformierte $\subseteq$ die Form

$$
\widetilde{S}^{\prime \prime}=\left(\begin{array}{ccc}
1 & S_{12} & 0 \\
S_{12} & 1 & S_{12} \\
0 & S_{12} & 1
\end{array}\right),
$$

und es ist $\left|\mathbb{S}^{\prime \prime}\right|=0$ für $S_{12}=1 / \sqrt{2}$. Bei gleicher Transformation der $\mathfrak{5}$-Matrix muß aber - wie oben gezeigt - auch $\left|\mathfrak{S C}^{\prime \prime}\right|=0$ sein, so daß auch in diesem Fall gemäß (8) und (15) die Eigenwerte endlich bleiben. In der Praxis verwendet man aber an 
Stelle von $\mathfrak{S}^{\prime \prime}$ die amputierte Form (16), deren Determinante für $S_{12}=1 / \sqrt{2}$ nicht verschwindet. Daher treten in der für diesen Fall zuständigen Energieformel (2) unendliche Energiewerte auf. Das gleiche ist auch der Fall, wenn man die $H_{i k}$ empirisch bestimmt und dabei deren Abhängigkeit von den Kernkoordinaten nicht Rechnung trägt, wie es in der Literatur gelegentlich geschieht.

\title{
Die Wärmeleitfähigkeit von gasförmigen Para-Ortho-Wasserstoffgemischen bei $20^{\circ} \mathrm{K}$
}

\author{
Von K. Heinzinger * A. Klemm und L. Waldmann \\ Aus dem Max-Planck-Institut für Chemie (Otto-Hahn-Institut), Mainz \\ (Z. Naturforschg. 16 a, 1338-1342 [1961] ; eingegangen am 6. September 1961)
}

Werner Heisenberg zum 60. Geburtstag gewidmet

\begin{abstract}
Mit einer Differentialmethode wurden bei $20^{\circ} \mathrm{K}$ die relativen Differenzen der Wärmeleitfähigkeiten von Para-Wasserstoff und verschiedenen Gemischen von Para- und Ortho-Wasserstoff gemessen. Es ergab sich, daß Para-Wasserstoff eine um $(5,84 \pm 0,30) \%$ größere Wärmeleitfähigkeit besitzt als Normal-Wasserstoff, und daß die Wärmeleitfähigkeit der Gemische als Funktion des OrthoWasserstoffgehalts negative zweite Ableitung hat. Das Ergebnis stimmt mit entsprechenden Zähigkeitsmessungen von BECKER und STEHL überein und hat noch keine vollständige theoretische Erklärung gefunden.
\end{abstract}

Para- und Ortho-Wasserstoff, auf deren Existenz erstmals Heisenberg in seiner grundlegenden Arbeit über Mehrkörperprobleme in der Quantenmechanik hingewiesen hatte ${ }^{1}$, befinden sich bei $20^{\circ} \mathrm{K}$ praktisch in ihren Grundzuständen, in denen $p-\mathrm{H}_{2}$ nicht rotiert und $o-\mathrm{H}_{2}$ den inneren Bahndrehimpuls $\hbar$ besitzt. Nimmt man an, daß die Wechselwirkungsenergie zweier $\mathrm{H}_{2}$-Moleküle, gleich welcher Art, ein und dieselbe Funktion nur des Abstandes der Molekülschwerpunkte ist, also nicht von den Bahndrehimpulsen und den Kernspins abhängt, so unterscheidet sich ein Zweierstoß im $p-\mathrm{H}_{2}$ von einem im $o-\mathrm{H}_{2}$ nur durch die Symmetrieforderungen, welche man an die jeweilige Zweiteilchen-Wellenfunktion zu stellen hat. Dieser Symmetrieunterschied allein sollte zu einem Unterschied der Transportkoeffizienten beider Gase bei tiefen Temperaturen führen. Auf die Möglichkeit dieses rein quantenmechanischen Effekts wurde von theoretischer Seite schon vor langem hingewiesen ${ }^{2}$.

Ältere Messungen bei $20^{\circ} \mathrm{K}$ hatten weder für die Zähigkeit ${ }^{3}$, noch für die Wärmeleitfähigkeit ${ }^{4,5}$ von $p-\mathrm{H}_{2}$ und Normalwasserstoff $\left(25 \% p-\mathrm{H}_{2}, 75 \% o-\mathrm{H}_{2}\right)$ einen Unterschied ergeben. Dabei gelangten allerdings die zu untersuchenden Gase nacheinander in

* Dissertation Mainz 1961 (D 77).

1 W. Heisenberg, Z. Phys. 41, 239 [1927].

2 O. Halpern u. E. Gwathmey, Phys. Rev. 52, 944 [1937].

3 P. Harteck u. H. W. Schmidt, Z. phys. Chem. B 21, 447 [1933]. die gekühlte Kapillare bzw. Wärmeleitfähigkeitszelle, was die Meßgenauigkeit wegen der zeitlichen Inkonstanz der Temperatur beeinträchtigte. Offenbar muß sich die Meßgenauigkeit durch Anwendung einer Differentialmethode, bei der zwei möglichst gleich gebaute Kapillaren bzw. Wärmeleitfähigkeitszellen im Kältebad nebeneinander und gleichzeitig mit den zu vergleichenden Gasen beschickt werden, erheblich steigern lassen.

Zähigkeitsmessungen, die mit einer solchen Differentialmethode ausgeführt wurden ${ }^{6}$, ergaben, daß $p$ - $\mathrm{H}_{2}$ bei $20^{\circ} \mathrm{K}$ eine um $5,6 \%$ größere Zähigkeit besitzt als Normal-Wasserstoff, und daß die Kurve, welche die Zähigkeit als Funktion des $o-\mathrm{H}_{2}$-Gehaltes darstellt, eine negative zweite Ableitung hat. In der vorliegenden Arbeit wird über entsprechende Messungen der Wärmeleitfähigkeit berichtet. Wie schon kurz mitgeteilt ${ }^{7}$, stimmen die Ergebnisse dieser Messungen gut mit denen der Zähigkeitsmessungen überein.

\section{Die Apparatur}

Abb. 1 zeigt den Plan der Apparatur. Wasserstoff aus einer Stahlflasche gelangt über eine Kapillare in den mit flüssigem Wasserstoff gekühlten und mit Aktiv-

\footnotetext{
4 J. B. UbBink, Physica 14, 165 [1948].

5 L. Waldmann u. E. W. Becker, Z. Naturforschg. 3 a, 180 [1948].

6 E. W. Becker u. O. Stehl, Z. Phys. 133, 615 [1952].

7 K. Heinzinger, Z. Naturforschg. 15 a, 1022 [1960].
} 